\title{
Proposal for ultrafast switching of ferroelectrics using midinfrared pulses
}

\author{
Alaska Subedi \\ Max Planck Institute for the Structure and Dynamics of Matter, Luruper Chaussee 149, 22761 Hamburg, Germany
}

(Received 30 October 2015; published 31 December 2015)

\begin{abstract}
I propose a method for ultrafast switching of ferroelectric polarization using midinfrared pulses. This involves selectively exciting the highest frequency $A_{1}$ phonon mode of a ferroelectric material with an intense midinfrared pulse. Large amplitude oscillations of this mode provides a unidirectional force to the lattice such that it displaces along the lowest frequency $A_{1}$ phonon mode coordinate because of a nonlinear coupling of the type $g Q_{\mathrm{P}} Q_{\mathrm{IR}}^{2}$ between the two modes. First-principles calculations show that this coupling is large in perovskite transition-metal oxide ferroelectrics, and the sign of the coupling is such that the lattice displaces in the switching direction. Furthermore, I find that the lowest frequency $A_{1}$ mode has a large $Q_{\mathrm{P}}^{3}$ order anharmonicity, which causes a discontinuous switch of electric polarization as the pump amplitude is continuously increased.
\end{abstract}

DOI: 10.1103/PhysRevB.92.214303

PACS number(s): 77.80.Fm, 78.20.Bh, 63.20.Ry, 78.47.J-

\section{INTRODUCTION}

Ultrafast switching of polarization in ferroelectrics is of great interest for potential application in nonvolatile memory devices. FLASH memories, which at present are the most commonly used nonvolatile memory devices, have an operating speed of milliseconds. Because of their slow speed, they are not considered as candidates for future memory applications [1]. Other emerging nonvolatile memory technologies that utilize phase or resistance change have write and erase times of nanoseconds. Therefore, development of a switching mechanism at subpicosecond time scales has the potential to revolutionize the field.

Nondestructive readout of the electric polarization at subpicosecond time scales has recently been demonstrated by analyzing the $\mathrm{THz}$ pulse waveforms radiated after illumination of a ferroelectric sample by femtosecond laser pulses at optical wavelengths $[2,3]$. This makes ferroelectric materials an exciting prospect for memory applications if switching can be achieved at similarly ultrashort time scales.

Ferroelectric materials exhibit remnant polarization even at zero external electric field because the cations and anions in these materials are asymmetrically displaced in the equilibrium structure. To switch the polarization, the relative displacement between the cations and anions need to be reversed. This can be achieved by applying a (quasi)static electric field because such an electric field imparts a unidirectional force to the cations and anions. An arbitrary light pulse, whose oscillating electric field integrates to zero by definition, imparts a zero total force to the electric dipole present in the material. Therefore, an ultrashort light pulse cannot in general be used to switch the polarization of a ferroelectric material. Nevertheless, there have been several proposals for switching the polarization of ferroelectric materials using ultrashort light pulses by controlling their soft phonon modes [4,5].

In this paper I propose a method for using midinfrared pulses that are resonant with the highest frequency infraredactive phonon mode of a perovskite transition-metal oxide ferroelectric to switch its polarization. This involves controlling the dynamical degrees of freedom of the lattice and requires four main ingredients. First, I notice that there always exists a low frequency fully symmetric $A_{1}$ phonon mode in the ferroelectric phase that involves the motion of the cations and anions of the material in a way that changes the electric polarization. Second, I find that this phonon mode couples to the highest frequency infrared-active $A_{1}$ phonon mode of the material with a large $g Q_{\mathrm{P}} Q_{\mathrm{IR}}^{2}$ coupling, where $g$ is the coupling constant and $Q_{\mathrm{P}}$ and $Q_{\mathrm{IR}}$ are the normal mode coordinates of the lowest frequency and highest frequency $A_{1}$ normal mode coordinates, respectively. Third, I find from first-principles calculations that the sign of the coupling is such that the excitation of the highest frequency $A_{1}$ mode provides a displacive force along the $Q_{\mathrm{P}}$ normal mode coordinate in the direction that switches the polarization. Fourth, I find that the $Q_{\mathrm{P}}$ mode has a strong $Q_{\mathrm{P}}^{3}$ order anharmonicity, which facilitates an abrupt switch of electric polarization as the $Q_{\mathrm{IR}}$ amplitude is continuously increased. Coherent displacement along Raman mode coordinates utilizing nonlinear phonon couplings by resonantly exiting the highest frequency infrared mode of various centrosymmetric oxides has previously been demonstrated [6-9]. Therefore, the method proposed here to switch ferroelectric polarization at ultrafast time scales using midinfrared pulses is experimentally feasible.

\section{COMPUTATIONAL DETAILS}

I illustrate the proposed mechanism for the case of $\mathrm{PbTiO}_{3}$. The phonon frequencies and eigenvectors and the nonlinear couplings between two phonon modes were obtained using density functional theory calculations with plane-wave basis sets and projector augmented wave pseudopotentials [10,11] as implemented in the VASP software package [12]. The interatomic force constants were calculated using the frozenphonon method [13], and the PHONOPY software package was used to calculate the phonon frequencies and eigenvectors [14]. Total energy calculations were then performed as a function of the lowest frequency $Q_{\mathrm{P}}$ and high-frequency $Q_{\mathrm{IR}}$ coordinates to obtain energy surfaces. The nonlinear couplings between the two modes were obtained by fitting the calculated energy surface to the polynomial shown in Eq. (1).

I used the experimental values of $a=3.9039$ and $c=$ $4.1348 \AA$ for the tetragonal lattice parameters but relaxed the atomic positions. The calculations were performed within the local density approximation. A cutoff of $600 \mathrm{eV}$ was used for the plane-wave basis set expansion, and an $8 \times 8 \times 8 k$-point grid was used in the Brillouin zone integration. 
TABLE I. The zone-center phonon frequencies and their irreducible representation and optical activity of ferroelectric $\mathrm{PbTiO}_{3}$

\begin{tabular}{lcc}
\hline \hline Frequency $\left(\mathrm{cm}^{-1}\right)$ & Irrep & Optical activity \\
\hline 80 & $E$ & IR + Raman \\
149 & $A_{1}\left(Q_{\mathrm{P}}\right)$ & $\mathrm{IR}+$ Raman \\
187 & $E$ & $\mathrm{IR}+$ Raman \\
273 & $E$ & $\mathrm{IR}+$ Raman \\
290 & $B_{1}$ & Raman \\
356 & $A_{1}$ & $\mathrm{IR}+$ Raman \\
491 & $E$ & $\mathrm{IR}+$ Raman \\
655 & $A_{1}\left(Q_{\mathrm{IR}}\right)$ & $\mathrm{IR}+$ Raman \\
\hline \hline
\end{tabular}

\section{RESULTS AND DISCUSSIONS}

The ferroelectric phase of $\mathrm{PbTiO}_{3}$ exists in the $\mathrm{P} 4 \mathrm{~mm}$ structure with one formula unit per unit cell. This gives rise to 12 zone-center optical normal modes with the decomposition $\Gamma_{\text {optic }}=3 A_{1}+B_{1}+4 E$. The $A_{1}$ and $E$ modes are both Raman and infrared active, whereas the $B_{1}$ is only Raman active. The calculated zone center phonon frequencies and their symmetries are given in Table I. I find that the coupling between the lowest frequency $E$ and the highest frequency $A_{1}$ mode is weak. Therefore, I focus on the coupled dynamics of the lowest and highest frequency $A_{1}$ modes.

The atomic displacement pattern of the lowest frequency $A_{1}$ mode (denoted by $Q_{\mathrm{P}}$ ) is shown in Fig. 1(a) with $z$ axis chosen as the polarization axis. A finite magnitude of this mode involves the motion of $\mathrm{Pb}^{2+}$ and $\mathrm{O}^{2-}$ ions along the $z$ axis in the opposite direction, and a displacement of the lattice along the coordinate of this mode modifies the electric polarization of the material. In the convention used in this paper, a large negative value of this normal mode coordinate would reverse the polarization. The arrows in Fig. 1(a) indicate the movements of ions for such a negative value of $Q_{\mathrm{P}}$. One can see that such a movement reverses the relative displacement between the $\mathrm{Pb}^{2+}$ and $\mathrm{O}^{2-}$ ions. However, it should be noted that a displacement along this mode does not bring the structure to the symmetrically equivalent ground state with opposite polarization because the eigenvector of this mode is not in general equal to the eigenvector of the unstable infrared mode of the paraelectric phase that is responsible for the ferroelectric instability. A further relaxation of the lattice, in addition to the

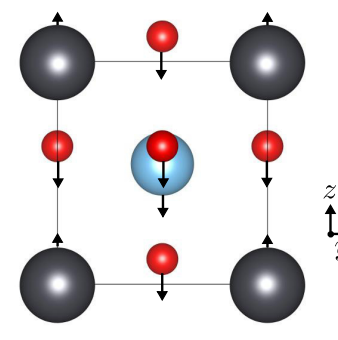

(a)

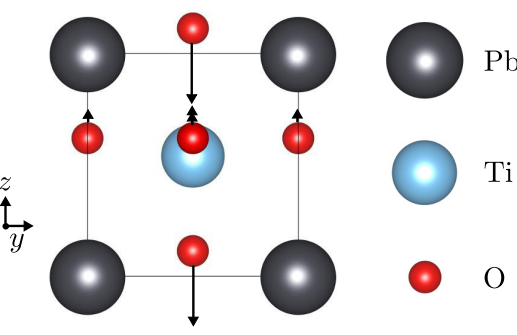

(b)
FIG. 1. (Color online) Displacement patterns of the (a) lowest frequency $\left(149 \mathrm{~cm}^{-1}\right) Q_{\mathrm{P}}$ and (b) highest frequency $\left(655 \mathrm{~cm}^{-1}\right) Q_{\mathrm{IR}}$ modes of the ferroelectric phase of $\mathrm{PbTiO}_{3}$. Both modes belong to the $A_{1}$ irreducible representation. large negative value of the $Q_{\mathrm{P}}$ coordinate that reverses the polarization, would take the structure to the symmetrically equivalent switched ground state. I confirmed this by starting with a structure that was displaced by a value of $-8 \AA \sqrt{\mathrm{amu}}$ along the $Q_{\mathrm{P}}$ coordinate and relaxing the atomic positions by minimizing the forces. I found that the structure indeed relaxes to the symmetrically equivalent switched phase rather than going back to the initial ferroelectric equilibrium state that was used as a starting point to displace along the $Q_{\mathrm{P}}$ coordinate.

Therefore, a coherent displacement of the lattice along this low frequency $Q_{\mathrm{P}}$ phonon mode coordinate is a viable route for ultrafast ferroelectric switching. In fact, Qi et al. have proposed a method for switching the polarization by driving large amplitude oscillations of this phonon mode using multiple $\mathrm{THz}$ pulses with an asymmetric electric field profile [5].

Here I propose a method of switching the polarization using a light pulse that does not directly drive the low frequency $Q_{\mathrm{P}}$ mode. Instead, this involves exciting the high frequency $Q_{\mathrm{IR}}$ infrared mode of the material [shown in Fig. 1(b)] by an intense midinfrared pulse that in turn provides a displacive force along the $Q_{\mathrm{P}}$ coordinate in the switching direction due to a nonlinear coupling of the type $g Q_{\mathrm{P}} Q_{\mathrm{IR}}^{2}$ between the two modes. Furthermore, the presence of a large $Q_{\mathrm{P}}^{3}$ order anharmonicity causes a sudden increase in the displacement along the $Q_{\mathrm{P}}$ coordinate as the $Q_{\mathrm{IR}}$ amplitude is continuously increased, and this causes an abrupt reversal of the electric polarization without the magnitude of the polarization going to zero.

I calculated the total energy as a function of the lowest frequency $Q_{\mathrm{P}}$ and highest frequency $Q_{\mathrm{IR}}$ infrared mode coordinates from first principles using density functional theory calculations. The calculated energy surface of ferroelectric $\mathrm{PbTiO}_{3}$ is shown in Fig. 2, and it fits the following expression:

$$
\begin{aligned}
V\left(Q_{\mathrm{P}}, Q_{\mathrm{IR}}\right)= & \frac{1}{2} \Omega_{\mathrm{P}}^{2} Q_{\mathrm{P}}^{2}+\frac{1}{2} \Omega_{\mathrm{IR}}^{2} Q_{\mathrm{IR}}^{2}+\frac{1}{3} a_{3} Q_{\mathrm{P}}^{3} \\
& +\frac{1}{4} a_{4} Q_{\mathrm{P}}^{4}+\frac{1}{3} b_{3} Q_{\mathrm{IR}}^{3}+\frac{1}{4} b_{4} Q_{\mathrm{IR}}^{4} \\
& +g Q_{\mathrm{P}} Q_{\mathrm{IR}}^{2}+h Q_{\mathrm{P}}^{2} Q_{\mathrm{IR}}+i Q_{\mathrm{P}}^{3} Q_{\mathrm{IR}} \\
& +j Q_{\mathrm{P}} Q_{\mathrm{IR}}^{3}+k Q_{\mathrm{P}}^{2} Q_{\mathrm{IR}}^{3}+l Q_{\mathrm{P}} Q_{\mathrm{IR}}^{4} .
\end{aligned}
$$

A fit of the above expression to the calculated energy surface determines $a b$ initio the nonlinear couplings between the two modes up to all significant orders of the two phonon coordinates. The values of the coefficients of the coupling terms obtained from such a fit are given in Table II. The calculated energy surface exhibits complex features, and this is due to the presence of both even and odd order nonlinearities. However, there are some salient features. When $Q_{\mathrm{IR}}=0$, the energy curve of the $Q_{\mathrm{P}}$ mode has one minimum at zero, as one would expect for a stable ground-state structure. The energy increases rapidly for positive values of $Q_{\mathrm{P}}$, but the increase is less rapid for negative values of $Q_{\mathrm{P}}$. In fact, for negative values of $Q_{\mathrm{P}}$, the slope of the energy curve has a minimum near $-5 \AA \sqrt{\mathrm{amu}}$, where the energy curve is shallow and the restoring force is small, before it shows an upturn around $-8 \AA \sqrt{\mathrm{amu}}$. This asymmetric nature of the energy curve of the $Q_{\mathrm{P}}$ mode is due to the presence of a large $a_{3} Q_{\mathrm{P}}^{3}$ term in the polynomial expression of the energy surface. The physical reason for the asymmetric nature of the $Q_{\mathrm{P}}$ energy curve is 

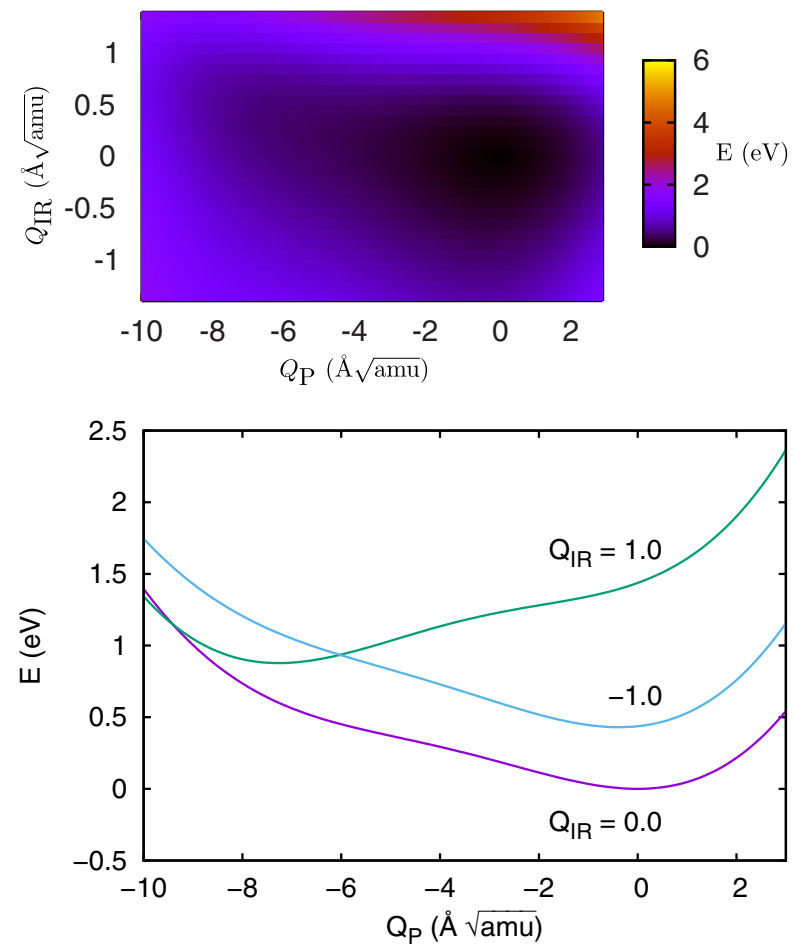

FIG. 2. (Color online) Total energy as a function of the $Q_{\mathrm{P}}$ and $Q_{\mathrm{IR}}$ normal mode coordinates of the ferroelectric $\mathrm{PbTiO}_{3}$. Top: Energy surface. Bottom: Few energy curves that illustrate the behavior of the $Q_{\mathrm{P}}$ mode as a function of $Q_{\mathrm{IR}}$ mode.

the presence of a state with reversed polarization near a $Q_{\mathrm{P}}$ value of $-8 \AA \sqrt{\mathrm{amu}}$ that is symmetrically equivalent to the ferroelectric state at a $Q_{\mathrm{P}}$ value of zero.

The energy surface is also asymmetric in the $Q_{\text {IR }}$ coordinate because of the presence of odd order terms. The presence of both even and odd order terms is consistent with the fact that this high frequency mode also has the $A_{1}$ representation that does not break any crystal symmetry and is both infrared and Raman active. Although the energy surface is asymmetric in the $Q_{\mathrm{IR}}$ coordinate, both positive and negative $Q_{\mathrm{IR}}$ dis-

TABLE II. The anharmonic terms and nonlinear couplings of the $Q_{\mathrm{P}}$ and $Q_{\mathrm{IR}}$ modes of ferroelectric $\mathrm{PbTiO}_{3}$ determined from a fit to the energy surface calculated from first principles.

\begin{tabular}{lr}
\hline \hline Coefficient & Value \\
\hline$a_{3}\left(\mathrm{meV} / \mathrm{amu}^{3 / 2} \AA^{-3}\right)$ & 21.80 \\
$a_{4}\left(\mathrm{meV} / \mathrm{amu}^{2} \AA^{-4}\right)$ & 1.89 \\
$b_{3}\left(\mathrm{meV} / \mathrm{amu}^{3 / 2} \AA^{-3}\right)$ & 1567.65 \\
$b_{4}\left(\mathrm{meV} / \mathrm{amu}^{2} \AA^{-4}\right)$ & 631.80 \\
$g\left(\mathrm{meV} / \mathrm{amu}^{3 / 2} \AA^{-3}\right)$ & 70.32 \\
$h\left(\mathrm{meV} / \mathrm{amu}^{3 / 2} \AA^{-3}\right)$ & 12.40 \\
$i\left(\mathrm{meV} / \mathrm{amu}^{2} \AA^{-4}\right)$ & -0.79 \\
$j\left(\mathrm{meV} / \mathrm{amu}^{2} \AA^{-4}\right)$ & 52.14 \\
$k\left(\mathrm{meV} / \mathrm{amu}^{5 / 2} \AA^{-5}\right)$ & 2.29 \\
$l\left(\mathrm{meV} / \mathrm{amu}^{5 / 2} \AA^{-5}\right)$ & 7.61 \\
\hline \hline
\end{tabular}

placements move the minimum of the $Q_{\mathrm{P}}$ coordinate towards the negative direction. A negative value of $Q_{\mathrm{IR}}$ displaces the lattice towards the negative $Q_{\mathrm{P}}$ direction by a modest amount. However, a positive value of $Q_{\text {IR }}$ does not continuously shift the energy minimum along the negative $Q_{\mathrm{P}}$ direction. It raises the energy curve near $Q_{\mathrm{P}} \approx 0$ and creates an energy minimum at a large negative value of the $Q_{\mathrm{P}}$ coordinate such that a state with reversed polarization is energetically favored. This abrupt shift of the minimum of the $Q_{\mathrm{P}}$ mode is due to the presence of a large $a_{3} Q_{\mathrm{P}}^{3}$ term.

If the oscillations along the $Q_{\mathrm{IR}}$ coordinate are integrated out, the average potential experienced by the lattice has a minimum at a negative value of the $Q_{\mathrm{P}}$ coordinate with the magnitude of the displacement along $Q_{\mathrm{P}}$ coordinate depending on the integration cutoff (i.e., the amplitude of the $Q_{\mathrm{IR}}$ oscillations). This is because the coupling constant $g$ of the term $Q_{\mathrm{P}} Q_{\mathrm{IR}}^{2}$ has the largest magnitude, and it implies that the lattice will experience a large unidirectional force $-\partial V / \partial Q_{\mathrm{P}}=-g Q_{\mathrm{IR}}^{2}$ along the $Q_{\mathrm{P}}$ coordinate when the $Q_{\mathrm{IR}}$ mode is being driven. Furthermore, a large $a_{3} Q_{\mathrm{P}}^{3}$ term ensures that the displacement of the lattice along the $Q_{\mathrm{P}}$ coordinate abruptly increases as the $Q_{\mathrm{IR}}$ amplitude is continuously increased, which causes the electric polarization to switch discontinuously.

We can achieve a better understanding of the dynamics of the lattice when the $Q_{\mathrm{IR}}$ mode is pumped externally by a midinfrared pulse by treating the $Q_{\mathrm{P}}$ and $Q_{\mathrm{IR}}$ modes as classical oscillators and studying their coupled equations of motion. In this picture, the two oscillators experience a force deriving from the calculated energy surface, and the $Q_{\mathrm{IR}}$ mode is additionally driven by a term $F(t)=F \sin (\Omega t) e^{-t^{2} / 2 \sigma^{2}}$, where $F, \sigma$, and $\Omega$ are the amplitude, width, and frequency of the midinfrared pulse, respectively. By treating the expression in Eq. (1) as the potential, the coupled equations motion are

$$
\begin{aligned}
\ddot{Q}_{\mathrm{IR}}+\Omega_{\mathrm{IR}}^{2} Q_{\mathrm{IR}}= & -b_{3} Q_{\mathrm{IR}}^{2}-b_{4} Q_{\mathrm{IR}}^{3}-2 g Q_{\mathrm{P}} Q_{\mathrm{IR}} \\
& -h Q_{\mathrm{P}}^{2}-i Q_{\mathrm{P}}^{3}-3 j Q_{\mathrm{P}} Q_{\mathrm{IR}}^{2} \\
& -3 k Q_{\mathrm{P}}^{2} Q_{\mathrm{IR}}^{3}-4 l Q_{\mathrm{P}} Q_{\mathrm{IR}}^{3}+F(t), \\
\ddot{Q}_{\mathrm{P}}+\Omega_{\mathrm{P}}^{2} Q_{\mathrm{P}}= & -a_{3} Q_{\mathrm{P}}^{2}-a_{4} Q_{\mathrm{P}}^{3}-g Q_{\mathrm{IR}}^{2} \\
& -2 h Q_{\mathrm{P}} Q_{\mathrm{IR}}-3 i Q_{\mathrm{P}}^{2} Q_{\mathrm{IR}}-j Q_{\mathrm{IR}}^{3} \\
& -2 k Q_{\mathrm{P}} Q_{\mathrm{IR}}^{3}-l Q_{\mathrm{IR}}^{4} .
\end{aligned}
$$

The results from numerical integration of the coupled equation of motions for different pump amplitudes are shown in Fig. 3. In these calculations, I have used a pump pulse with a symmetric Gaussian profile and a width of $\sigma=250 \mathrm{fs}$, which correspond to typical pump pulses used in midinfrared excitations $[6,7,9]$. A pump frequency of $\Omega=1.03 \Omega_{\mathrm{IR}}$ was used in the simulations, which is chosen to be slightly offresonance with the frequency of the $Q_{\text {IR }}$ mode to demonstrate that this method is efficacious even if the pump pulse is not precisely resonant with the $Q_{\mathrm{IR}}$ mode.

Even for small pump amplitudes that cause a change in the Ti-apical $\mathrm{O}$ distances of a few percent along the $Q_{\mathrm{IR}}$ coordinate, the $Q_{\mathrm{P}}$ mode oscillates at a displaced position in the negative $Q_{\mathrm{P}}$ direction [see the left panel of Fig. 3(a)]. This is consistent with the analysis of the $g Q_{\mathrm{P}} Q_{\mathrm{IR}}^{2}$ coupling presented in Refs. [6,8]. In Ref. [8] it was shown that the $Q_{\mathrm{P}}$ mode 

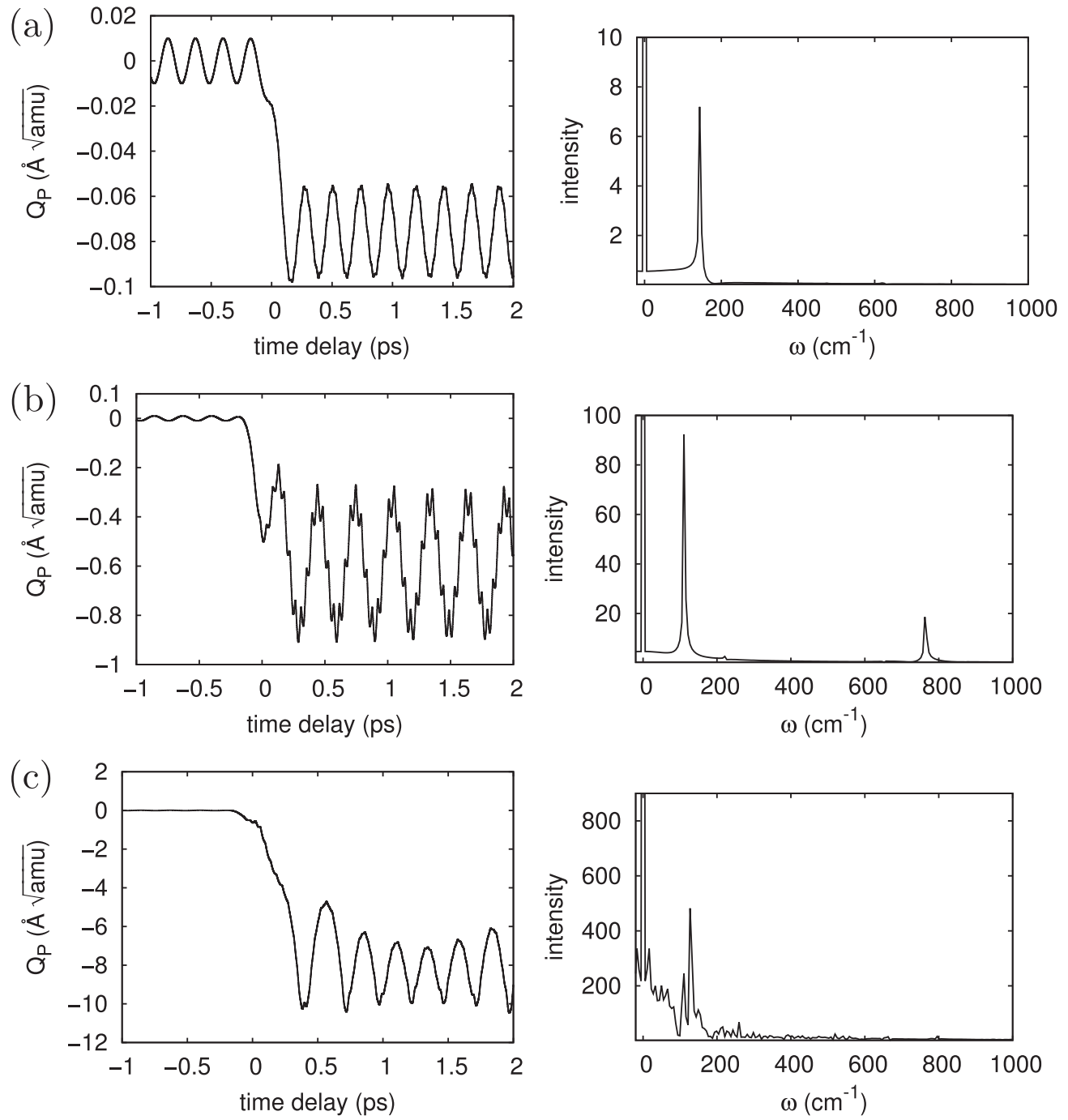

FIG. 3. Dynamics of the $Q_{\mathrm{P}}$ mode for three different pump amplitudes. Left panels: Displacements along $Q_{\mathrm{P}}$ coordinate as function of time delay. Right panels: Fourier transform of the positive time delay oscillations.

experiences an effective force $-g Q_{\mathrm{IR}}^{2} \propto-g F^{2} \Omega_{\mathrm{IR}}^{2} \sigma^{6}(1-$ $\left.\cos 2 \Omega_{\mathrm{IR}} t\right)$ when the $Q_{\mathrm{IR}}$ mode is pumped by an external driving term $F(t)$, and the time average of this forcing field has a rectified nonzero value. The oscillation of the $Q_{\mathrm{P}}$ mode at a rectified position is also seen in the Fourier transform of the time evolution of the $Q_{\mathrm{P}}$ mode at positive time delays as shown in the right panel of Fig. 3(a). The Fourier transform shows a peak at zero frequency that is due to the displacement of the lattice along the $Q_{\mathrm{P}}$ coordinate. In addition, there is a peak at $\Omega_{\mathrm{P}}$ and a negligible presence of higher harmonics, which shows that the dynamics of the coupled oscillators are determined by the $g Q_{\mathrm{P}} Q_{\mathrm{IR}}^{2}$ term, and other nonlinearities only play a marginal role at small pump amplitudes. At small pump amplitudes, the displacement along the negative direction in the $Q_{\mathrm{P}}$ coordinate is small. Therefore, although the electric polarization is reduced, the reversal of the polarization has not occurred.

It is also noteworthy that the cubic terms in the energy potential $a_{3} Q_{\mathrm{P}}^{3}$ and $b_{3} Q_{\mathrm{IR}}^{3}$ are large. These also impart unidirectional forces $-\partial V / \partial Q_{\mathrm{P}}=-a_{3} Q_{\mathrm{P}}^{2}$ and $-\partial V / \partial Q_{\mathrm{IR}}=$ $-b_{3} Q_{\mathrm{IR}}^{2}$ to their respective coordinates when the amplitude of the oscillations are large, and the lattice will be rectified along these coordinates for the reasons described in the previous paragraph. From my first-principles calculations of the energy surface, I find that the coefficients of these terms are such that the lattice is rectified along the direction that reverses the electric polarization.

As the pump amplitude is increased, the effects of the above-mentioned nonlinearities start to become noticeable. The oscillations of the $Q_{\mathrm{P}}$ mode start to show higher frequency components due to the presence of various nonlinear terms. Further increase of the pump amplitude takes the dynamics to a highly nonlinear regime. In this regime, the frequency at which the $Q_{\mathrm{P}}$ mode oscillates also changes. Interestingly, there are two different mechanisms that change the effective frequency of the $Q_{\mathrm{P}}$ mode. First, a large amplitude oscillation of the $Q_{\mathrm{P}}$ mode causes a change in the effective spring constant due to the $\frac{1}{4} a_{4} Q_{\mathrm{P}}^{4}$ nonlinearity in the energy potential. In the equation of motion, this nonlinearity acts to modify the frequency of the $Q_{\mathrm{P}}$ mode with a term $-\partial V / \partial Q_{\mathrm{P}}=-a_{4} Q_{\mathrm{P}}^{3}$. The effective frequency $\Omega_{\mathrm{P}}^{\text {eff }} \rightarrow \Omega_{\mathrm{P}}^{2}\left[1+a_{4} Q_{\mathrm{P}}^{2}(t) / \Omega_{\mathrm{P}}^{2}\right]$ changes because the time-averaged $\Omega_{\mathrm{P}}^{\text {eff }}$ has a value different from $\Omega_{\mathrm{P}}$ when the $Q_{\text {P }}$ amplitude is large. The rectification of the lattice along 
the $Q_{\mathrm{P}}$ and $Q_{\mathrm{IR}}$ coordinates provides the second cause for the modification of the effective frequency. For example, the rectification along the $Q_{\mathrm{P}}$ coordinate changes the effective frequency to $\Omega_{\mathrm{P}}^{\text {eff }} \rightarrow \Omega_{\mathrm{P}}^{2}\left[1+a_{3} Q_{\mathrm{P}}(t) / \Omega_{\mathrm{P}}^{2}\right]$ due to the $a_{3} Q_{\mathrm{P}}^{3}$ term in the energy potential. The $h Q_{\mathrm{P}}^{2} Q_{\mathrm{IR}}$ term in the energy potential similarly changes the effective frequency as $\Omega_{\mathrm{P}}^{\mathrm{eff}} \rightarrow$ $\Omega_{\mathrm{P}}^{2}\left[1+2 h Q_{\mathrm{IR}}(t) / \Omega_{\mathrm{P}}^{2}\right]$. Figure $3(\mathrm{~b})$ shows the results of the numerical integration of the equations of motion for a pump amplitude that rectifies the lattice close to a point where the polarization switches. Near the polarization reversal, the slope of the potential for the $Q_{\mathrm{P}}$ mode is less steep, and the $Q_{\mathrm{P}}$ mode oscillates at a smaller frequency. In this regime, the pumped $Q_{\text {IR }}$ mode is oscillating at a displaced position with an amplitude that changes the two Ti-apical $\mathrm{O}$ bond lengths by 0.4 and $0.7 \AA$. These are large amplitude oscillations, but they are comparable to the change in the Ti-apical $\mathrm{O}$ distance of $0.6 \AA$ when a polarization switch occurs.

Figure 3(c) illustrates the case where the lattice moves to a far distance in the negative direction along the $Q_{\mathrm{P}}$ coordinate, and this signals that the polarization has been switched. The pump strength used in this instance causes the $Q_{\mathrm{IR}}$ mode to oscillate at a displaced position with an amplitude that changes the two Ti-apical $\mathrm{O}$ bond lengths by 0.5 and 0.8 $\AA$. In this regime, the oscillations about the displaced position exhibit a strong nonlinear behavior with the presence of a wide range of frequency components. Nevertheless, the frequency component that has the largest spectral weight stiffens once the displacement along $Q_{\mathrm{P}}$ coordinate advances through the point of polarization reversal, although the frequency is still smaller than $\Omega_{\mathrm{P}}$.

I find that the displacement along the $Q_{\mathrm{P}}$ coordinate shows a sudden jump when the externally pumped $Q_{\mathrm{IR}}$ amplitude is continuously increased. This is consistent with the behavior of the energy potential discussed above where a large $a_{3} Q_{\mathrm{P}}^{3}$ term causes an abrupt change in the position of the minimum of the $Q_{\mathrm{P}}$ mode as $Q_{\mathrm{IR}}$ is continuously increased. As a function of the pump amplitude, the displacement along the $Q_{\text {P }}$ coordinate continuously increases from a value of zero to $\sim-1.5 \AA \sqrt{\mathrm{amu}}$. However, a further increase of the pump amplitude causes the $Q_{\mathrm{P}}$ mode to oscillate about a displaced position of $\sim-9.0 \AA \sqrt{\mathrm{amu}}$. This indicates that the electric polarization switches in an abrupt, discontinuous manner when the $Q_{\text {IR }}$ mode is externally pumped. Such a behavior can also be gleaned from the change in the frequency of the $Q_{\mathrm{P}}$ mode as the polarization reversal happens. I find that the frequency of the $Q_{\mathrm{P}}$ mode decreases by up to $60 \%$ as it is displaced along this coordinate. But it does not soften completely to zero as the polarization switch occurs and the frequency starts to increase again.

In the study presented here, nonlinear couplings between two phonon modes and their dynamics when the higher frequency mode is externally pumped has been used to predict that ferroelectric materials can be switched using midinfrared pulses. However, in real materials there are additional dynamical degrees of freedom, and this has two main implications. First, scattering with other degrees of freedom will cause the phonon modes to be damped. Therefore, the rectifying force along the $Q_{\mathrm{P}}$ coordinate exists only as long as the $Q_{\mathrm{IR}}$ mode is being externally pumped. Second, other degrees of freedom also respond to the displacement of the lattice along the $Q_{\mathrm{P}}$ coordinate. If the pump pulse is long enough, other degrees of freedom relax relative to the switched state, and this forms an energy barrier that prevents the lattice to move back to the initial state even in the absence of the pump. A more detailed theoretical study based on molecular dynamics simulations would be required to ascertain the time it would take to form the energy barrier.

As mentioned above, I performed a full relaxation of the lattice starting from a structure that corresponds to a $Q_{\mathrm{P}}$ displacement of $-8.0 \AA \sqrt{\mathrm{amu}}$ and found that the lattice indeed relaxes to the symmetrically equivalent switched state. The relaxation of the whole lattice to the symmetrically equivalent state with reversed polarization provides a mechanism for repeated switching because the lattice again experiences a unidirectional force in the switching direction along the $Q_{\mathrm{P}}$ coordinate when the lattice is excited anew by a midinfrared pulse.

In addition to $\mathrm{PbTiO}_{3}$, I investigated the nonlinear couplings between the lowest and highest frequency $A_{1}$ modes of $\mathrm{BaTiO}_{3}$ and $\mathrm{LiNbO}_{3}$. I find a large $g Q_{\mathrm{P}} Q_{\mathrm{IR}}^{2}$ coupling between the lowest frequency $A_{1}$ mode $\left(Q_{\mathrm{P}}\right)$ and the highest frequency $A_{1}$ mode $\left(Q_{\mathrm{IR}}\right)$ in these materials as well. The sign of the coupling is such that the electric polarization of these materials could also be switched by pumping the $Q_{\mathrm{IR}}$ mode with a midinfrared pulse. Therefore, the method illustrated here seems applicable in general to all perovskite transition-metal oxide ferroelectrics.

\section{SUMMARY}

In summary, I have illustrated that the polarization of $\mathrm{PbTiO}_{3}$ can be switched by exciting the highest frequency infrared active $A_{1}$ phonon mode of this material with a midinfrared pulse. A large amplitude oscillation of this mode provides a unidirectional force along the lowest frequency $A_{1}$ phonon mode coordinate due to a nonlinear coupling of the type $g Q_{\mathrm{P}} Q_{\mathrm{IR}}^{2}$. A displacement of the lattice along the $Q_{\mathrm{P}}$ coordinate changes the electric polarization and can bring the system near the symmetrically equivalent switched state. From my first-principles calculations, I find that sign of the coupling is such that the oscillations of the $Q_{\mathrm{IR}}$ mode displaces the lattice along the $Q_{\mathrm{P}}$ coordinate in the switching direction. I also find that the switching occurs discontinuously because of the presence of a large $a_{3} Q_{\mathrm{P}}^{3}$ term in the energy potential, which abruptly moves the minimum of the $Q_{\mathrm{P}}$ mode as the $g Q_{\mathrm{P}} Q_{\mathrm{IR}}^{2}$ term gets continuously larger.

In addition to $\mathrm{PbTiO}_{3}$, I find the presence of a similar $g Q_{\mathrm{P}} Q_{\mathrm{IR}}^{2}$ coupling and a large $a_{3} Q_{\mathrm{P}}^{3}$ anharmonicity in $\mathrm{BaTiO}_{3}$ and $\mathrm{LiNbO}_{3}$, and this type of nonlinear coupling seems to be universally present in perovskite transition-metal oxide ferroelectrics. Therefore, a selective excitation of the $Q_{\mathrm{IR}}$ mode using a midinfrared pulse can be a general method to switch the polarization of perovskite transition-metal oxide ferroelectrics.

\section{ACKNOWLEDGMENTS}

I am indebted to Yannis Laplace for valuable discussions. I also acknowledge Antoine Georges, Roman Mankowsky, Srivats Rajasekaran, and Andrea Cavalleri for helpful comments and discussions. 
[1] D. S. Jeong, R. Thomas, R. S. Katiyar, J. F. Scott, H. Kohlstedt, A. Petraru, and C. S. Hwang, Rep. Prog. Phys. 75, 076502 (2012).

[2] K. Takahashi, N. Kida, and M. Tonouchi, Phys. Rev. Lett. 96, 117402 (2006).

[3] D. Talbayev, S. Lee, S.-W. Cheong, and A. J. Taylor, Appl. Phys. Lett. 93, 212906 (2008).

[4] S. Fahy and R. Merlin, Phys. Rev. Lett. 73, 1122 (1994).

[5] T. Qi, Y.-H. Shin, K.-L. Yeh, K. A. Nelson, and A. M. Rappe, Phys. Rev. Lett. 102, 247603 (2009).

[6] M. Först, C. Manzoni, S. Kaiser, Y. Tomioka, Y. Tokura, R. Merlin, and A. Cavalleri, Nat. Phys. 7, 854 (2011).

[7] M. Först, R. Mankowsky, H. Bromberger, D. M. Fritz, H. Lemke, D. Zhu, M. Chollet, Y. Tomioka, Y. Tokura, R. Merlin,
J. P. Hill, S. L. Johnson, and A. Cavalleri, Solid State Commun. 169, 24 (2013).

[8] A. Subedi, A. Cavalleri, and A. Georges, Phys. Rev. B 89, 220301 (2014).

[9] R. Mankowsky, A. Subedi, M. Först, S. O. Mariager, M. Chollet, H. T. Lemke, J. S. Robinson, J. M. Glownia, M. P. Minitti, A. Frano et al., Nature (London) 516, 71 (2014).

[10] P. E. Blöchl, Phys. Rev. B 50, 17953 (1994).

[11] G. Kresse and D. Joubert, Phys. Rev. B 59, 1758 (1999).

[12] G. Kresse and J. Furthmüller, Phys. Rev. B 54, 11169 (1996).

[13] K. Parlinski, Z-.Q. Li, and Y. Kawazoe, Phys. Rev. Lett. 78, 4063 (1997).

[14] A. Togo, F. Oba, and I. Tanaka, Phys. Rev. B 78, 134106 (2008). 